\title{
Coverage dependence of the Fe-induced Fermi-level shift and the two-dimensional electron gas on $\operatorname{InAs}(110)$
}

\author{
M. Morgenstern, M. Getzlaff, D. Haude, and R. Wiesendanger \\ Institute of Applied Physics and Microstructure Research Center, Hamburg University, Jungiusstraße 11, D-20355 Hamburg, Germany \\ R. L. Johnson \\ I. Institute of Experimental Physics, Hamburg University, Luruper Chausee 149, D-22671 Hamburg, Germany
}

(Received 17 November 1999; revised manuscript received 18 January 2000)

\begin{abstract}
The coverage dependence of the Fe-induced Fermi-level shift on $p$ - and $n$-InAs(110) was investigated by angle-resolved photoelectron spectroscopy at $300 \mathrm{~K}$. The Fermi-level position was found to be coverage dependent, exhibiting a maximum at $300 \mathrm{meV}$ above the conduction-band minimum. The coverage dependence is explained by the surface doping model, if inhomogeneities in the Fe-adatom distribution and the resulting ionization probabilities are taken into account. The Fe-adatom distribution is determined by scanning tunneling microscopy. Photoemission spectra provided direct evidence of a two-dimensional electron gas at the Fe-covered surface.
\end{abstract}

\section{INTRODUCTION}

The interface of III/V semiconductors with adsorbates of different kinds has been investigated extensively since the 1970s, triggered by the increasing technological interest in these materials. Recently, considerable attention was focused on band offsets and the position of the Fermi level $\left(E_{F}\right)$ at the interface. ${ }^{1}$ For large gap materials it is found that $E_{F}$ is pinned in the band gap. ${ }^{1}$ For small gap materials like InAs and InSb, the interface Fermi level is in the conduction band of the semiconductor. ${ }^{2,3}$ In particular, on $\operatorname{InAs}(110), E_{F}$ is shifted $0.1-0.6 \mathrm{eV}$ above the conduction band minimum $(\mathrm{CBM})$. This is found for very different adsorbates such as $\mathrm{H}, \mathrm{O}, \mathrm{N}, \mathrm{Cl}, \mathrm{Ag}, \mathrm{Au}, \mathrm{Ga}, \mathrm{Cu}, \mathrm{Cs}, \mathrm{Na}$, and $\mathrm{Sb}^{4-14}$ With adequate doping of the semiconductor, the corresponding surface band bending leads to a two-dimensional electron gas (2DEG) at the surface. ${ }^{15}$ Pump and probe experiments performed on these type of 2DEG's revealed that the $2 \mathrm{DEG}$ levels exhibit a lifetime as large as 100 ps, which indicates that the mobility of the $2 \mathrm{DEG}$ is quite high. ${ }^{16}$ The existence of a highly mobile 2DEG in combination with the large Rashba coefficient of InAs (Ref. 17) makes the ferromagnet/ InAs(110) system an interesting candidate for magnetoelectronic devices such as spin transistors. ${ }^{18}$

However, for ferromagnetic adsorbates on $\operatorname{InAs}(110)$ an $E_{F}$ shift above the CBM has not been demonstrated. Here we present angle-resolved photoemission spectra (ARUPS) of the system $\mathrm{Fe} / \mathrm{InAs}(110)$ at different coverages on both $n$ and $p$-InAs(110). We find that $E_{F}$ shifts up to $300 \mathrm{meV}$ into the conduction band for the two differently doped samples. The $n$-doped sample exhibits a 2DEG probably consisting of two subbands. The Fe-coverage necessary to obtain the maximum $E_{F}$ shift depends on the dopant concentration consistent with the surface doping model. ${ }^{19}$ However, inhomogenities in the spatial distribution of the Fe atoms have to be taken into account to explain the coverage dependence quantitatively.

\section{EXPERIMENT}

The $n$ - and $p$-type InAs samples are doped with $\mathrm{S}$ and Zn, respectively $\left(N_{D}=1.1 \times 10^{16} \mathrm{~cm}^{-3}, N_{A}=3 \times 10^{18} \mathrm{~cm}^{-3}\right)$. Clean and smooth (110) surfaces were prepared by cleaving the InAs samples in UHV $\left(2 \times 10^{-10}\right.$ mbar $) .^{20} \mathrm{Fe}$ is deposited from an $e$-beam evaporator with a flux of $0.1 \% \mathrm{ML} / \mathrm{s}$ for coverages up to $8 \% \mathrm{ML}$ and $2 \% \mathrm{ML} / \mathrm{s}$ at higher coverages. The sample temperature during deposition was $300 \mathrm{~K}$. The flux was determined by a quartz-crystal microbalance and specified in terms of the unit cell of InAs(110) (100\% ML means one Fe atom per unit cell). A set of up to seven photoemission spectra was taken at each coverage with the VG ADES 400 spectrometer using $p$-polarized light from the Seya-Namioka beamline at HASYLAB/DESY for photon energies $h \nu=10-30 \mathrm{eV}$. The monochromator used is not suitable for measurements of the In $4 d$ core level under surface-sensitive conditions. Thus we decided to concentrate on the valence band of $\operatorname{InAs}(110)$. The orientation of the sample with respect to the spectrometer was determined by low-energy electron diffraction. The energy resolution of the monochromator (depending on photon energy) was always below $20 \mathrm{meV}$. The energy resolution of the electron analyzer depends on $E_{\text {pass }}(1.5-4 \mathrm{eV})$ and is $\Delta E=0.02$ $\times E_{\text {pass }}$. The geometrical acceptance angle of the spectrometer is $\pm 2.2^{\circ}$. All of the spectra were recorded within 10150 min after cleavage or Fe deposition. The spectra used to determine the valence-band shifts with respect to $E_{F}$ were taken at similar times after deposition. The Fermi energy was determined on metallic $\mathrm{TbNi}_{2} \mathrm{~B}_{2} \mathrm{C}$.

The scanning tunneling microscopy measurements were performed independently in a UHV low temperature scanning tunneling microscopy (STM) apparatus described elsewhere. ${ }^{20}$ For these measurements Fe was deposited at room temperature on an in situ cleaved $n$-InAs(110) sample $\left(N_{D}=2.0 \times 10^{16} \mathrm{~cm}^{-3}\right)$ using an $e$-beam evaporator. The sample was cooled down and reached the imaging temperature of $8 \mathrm{~K}$ about 5 min after Fe deposition. 


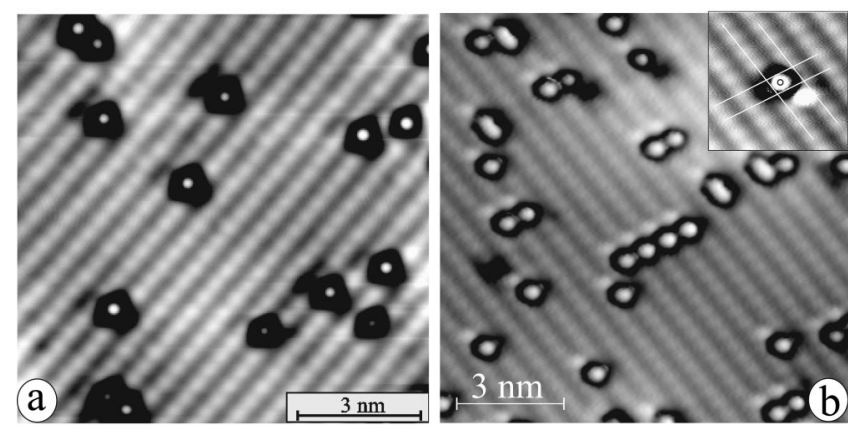

FIG. 1. (a) STM (constant current) image of 3\% ML Fe on $n$-InAs(110): $U=50 \mathrm{mV}, I=200 \mathrm{pA}$, and $T=8 \mathrm{~K}$. (b) STM (constant current) image of 7.5\% ML Fe on $n$-InAs(110): $U=50 \mathrm{mV}$, $I=100 \mathrm{pA}$, and $T=6 \mathrm{~K}$. Inset: magnification from (b). Lines indicate visible atomic rows, and circle indicate the visible center of the Fe atom. The two images (a),(b) are recorded on different samples, obviously oriented differently, with different microtips.

\section{RESULTS}

Figure 1 shows two STM images of the InAs(110) surface after deposition of 3\% (a) and $7.5 \% \mathrm{Fe}$ (b), respectively. The atomic rows of one type of atoms of the $\operatorname{InAs}(110)$ surface are visible, running diagonally through the image. ${ }^{21}$ The bright dots surrounded by a black area represent the Fe atoms. The slightly different shapes of the protrusion and the dark areas in the different images as well as the additional bright spot at the upper right corner of the $\mathrm{Fe}$ atoms in Fig. 1(b) are attributed to the influence of different microtips. As indicated in the inset, the center of the protrusion is always located between two atomic rows of the substrate. It is $2 \AA$ away from one row and $4 \AA$ away from the other row. In the perpendicular direction, it is between two adjacent maxima inside the rows. This position corresponds to the expected position of the surface atoms that are not visible. Since we do not know which species is visible, ${ }^{22}$ we cannot give the bond site of Fe. However, we can conclude that all $\mathrm{Fe}$ atoms bond to the same site.

In addition to single Fe adatoms, dimers, trimers and tetramers are visible. By counting the number of adatoms, dimers, trimers, and tetramers of different shape (in several STM images), we evaluated the percentage of Fe atoms having one or two neighbors parallel or perpendicular to the substrate rows. At $3 \%$ coverage, $22 \pm+3 \%$ of the $\mathrm{Fe}$ atoms have one neighbor perpendicular to the rows, $2.5 \pm 1 \%$ have one neighbor parallel to the rows, and $2 \pm 1 \%$ have two neighbors. At $7.5 \%$ coverage the corresponding numbers are $35 \pm 3 \%$ (perpendicular), $13 \pm 2 \%$ (parallel) and $6 \pm 2 \%$ (twofold). The average number of neighbors perpendicular to the rows and the number of twofold bonded Fe atoms is much higher than would be expected from a statistical distribution (e.g., statistically only $6 \%$ of the Fe atoms would be expected to have a neighbor perpendicular to the rows at 3\% coverage). At $8 \mathrm{~K}$ diffusion was not observed. Thus, the bond formation must be due to diffusion taking place at 300 $\mathrm{K}$.

The number of neighbors perpendicular to the rows is larger than parallel to the rows. This means that the diffusion is anisotropic and the atoms move mainly parallel to the rows. Assuming that the diffusion barriers do not depend on

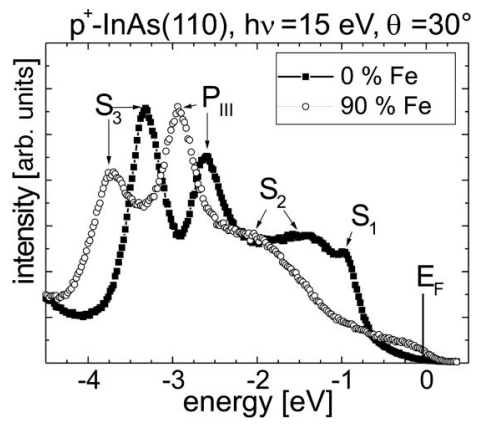

FIG. 2. ARUP spectrum of $0 \%$ and $90 \% \mathrm{ML} \mathrm{Fe}$ on $p^{+}-\operatorname{InAs}(110) ; h \nu=15 \mathrm{eV}, \theta=30^{\circ}$, and $E_{\text {pass }}=2.5 \mathrm{eV}$. Peaks are labeled according to Andersson and co-workers (Ref. 23).

neighbors more than one unit cell away from the moving atom and that dimer bonds are stable at $300 \mathrm{~K}$, we can estimate the jump rate of the $\mathrm{Fe}$ atoms. By counting all possible paths leading to bond formation for initially separate $\mathrm{Fe}$ atoms, we found that two jumps per Fe atom are sufficient to explain the number of bonds determined at both coverages. Thus, diffusion is slow at $300 \mathrm{~K}$ (about 1 jump every $2 \mathrm{~min}$ ), which means that the $\mathrm{Fe}$ atoms, at least at low coverage, are predominantely monomers and do not form large metallic islands.

Next we describe the angle-resolved photoemission results. Spectra of clean InAs(110) correspond closely to previous measurements. ${ }^{23}$ First we checked the influence of the residual gas on the ARUP spectra of clean and Fe-covered surfaces. All filaments are turned off during all measurements to prevent cracking or ionization of the residual gas molecules. It turned out that the spectra of clean and Fecovered $p$-InAs remained unchanged for more than two days, while the lower doped clean $n$-InAs exhibited an $E_{F}$ shift of $100 \mathrm{meV}$ already $3 \mathrm{~h}$ after cleavage, and the nearly full 300-meV shift after about $10 \mathrm{~h}$. The higher sensitivity of $n$-InAs(110) to residual gas molecules is in accordance with the fact that an $\mathrm{Fe}$ coverage of only $0.5 \%$ is sufficient to induce the maximum $E_{F}$ shift. In contrast, $4 \%$ coverage is needed on $p$-InAs(110) (see below). All measurements used for the evaluation of the $E_{F}$ shifts are obtained on time scales which were short compared to those required to produce contamination-induced shifts. Possible surface photovoltage effects can be ruled out with the help of the extensive tests performed by Aristov et al. ${ }^{8}$

For a determination of $E_{F}$ shifts we restrict ourselves to the (110) azimuth of the sample. The measured spectra include peaks related to three surface bands and four bulk bands. ${ }^{23}$ Figure 2 compares spectra measured directly after cleavage and after deposition of $90 \% \mathrm{Fe}$ on $p$-InAs(110). The peaks are labeled as proposed by Andersson and co-workers ${ }^{23}$ by $P_{n}$ and $S_{n}$ for transitions related to bulk and surface states, respectively. ( $P$ is used to denote primary cone emission.) First one observes that $P_{I I I}$ and $S_{3}$ are shifted about $400 \mathrm{meV}$ toward higher binding energies. Second, $S_{1}$ disappears completely, while $S_{2}$ and $S_{3}$ are reduced in intensity with respect to $P_{I I I}$. Third, spectral intensity appears up to $E_{F}$. The simultaneous shift of $P_{I I I}$ and $S_{3}$ is 

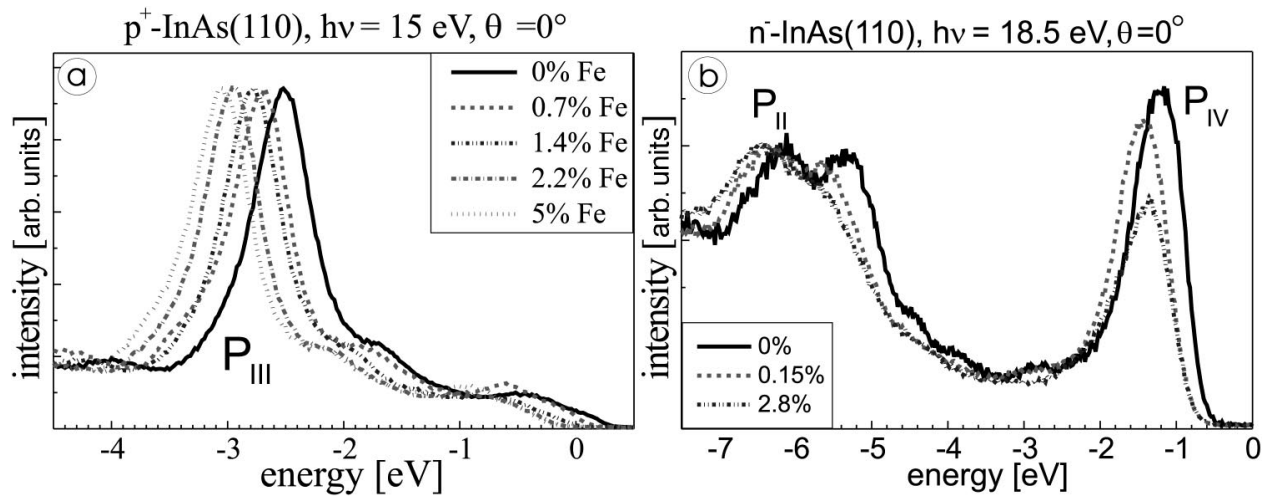

FIG. 3. (a) ARUP spectrum of $\mathrm{Fe} / p^{+}-\operatorname{InAs}(110)$ at different coverages: $h \nu=15 \mathrm{eV}, \theta=0^{\circ}$, and $E_{\text {pass }}=2.5 \mathrm{eV}$. (b) ARUP spectrum of $\mathrm{Fe} / n^{-}-\operatorname{InAs}(110)$ at different coverages; $h \nu=18.5 \mathrm{eV}, \theta$ $=0^{\circ}$, and $E_{\text {pass }}=2.5 \mathrm{eV}$. Peaks are labeled according to Ref. 23. equivalent to an $E_{F}$ shift of $400 \mathrm{meV}$ with respect to the valence band, i.e., $E_{F}$ is shifted above the CBM [the gap energy is $360 \mathrm{meV}$ (Ref. 24)]. The intensity change of the $S_{n}$ peaks shows that the surface states are more directly influenced by the adsorbate. In particular, the As dangling-bondrelated $S_{1}$ band is more sensitive to Fe deposition than the two backbond and in-plane bond-related surface bands $S_{2}$ and $S_{3} \cdot{ }^{23,25}$ We found that $S_{1}$ disappears at a coverage of $45 \%$. First indications of the existence of metallic Fe appear at a coverage of $15 \%$ as indicated by a measurable intensity at $E_{F}$.

Figure 3 shows two sets of photoemission spectra obtained at lower Fe coverages for $p^{+}$- and $n^{-}-\operatorname{InAs}(110)(+$ and - are used to indicate the different doping levels). The whole spectrum is shifted toward higher binding energies with respect to $E_{F}$. Consequently, the spectral features can be used to track the actual position of $E_{F}$ with respect to the valence band. It turned out that the $S_{n}$ peaks shift less than the $P_{n}$ peaks, especially at higher coverages. So only the sharp $P_{n}$ peaks in normal-emission spectra were used to determine $E_{F}$. Moreover, we selected peaks that are unaffected by surface contributions ${ }^{23}$ to avoid changes due to adsorbateinduced quenching of the surface peaks. Nevertheless some peaks, such as the $P_{I V}$ peak in Fig. 3(b), showed intensity changes while others [e.g., the $P_{I I I}$ peak in Fig. 3(a)] were constant up to coverages of $10 \%$, before they lost intensity. Since the $P_{I V}$ peak is important in a discussion of the Fermilevel shift, it should be noted that the observed intensity variations of this peak could be correlated with a slight misalignment of the sample relative to the incoming light and the electron analyzer. We found that the $P_{I I I}$ peak in Fig. 3 (a) is rather insensitive to misalignment up to $1^{\circ}$. The important conclusion is that the energy shifts due to mechanical misalignment were less than $50 \mathrm{meV}$.

Figure 4 shows the peak shifts obtained for different coverages and different selected $P_{n}$ peaks. All measured $P_{n}$ peaks confirm the trends shown in Fig. 4. The $P_{n}$ shift depends on the kinetic energy $E_{k i n}$ and thereby on the variation of the mean free path of the photoelectrons $(\lambda) .{ }^{1}$ Both are given in brackets. To obtain $E_{F}$ shifts from the data, this and an additional systematic errors have to be considered:

(1) The different values of $\lambda$ combined with the $z$ extension of the band bending imply that the measured peak energy is averaged over a $\lambda$-dependent part of the band bending. For $p^{+}$-InAs the parabolic band bending extends up to $13 \mathrm{~nm}$ into the bulk (see below). It is straightforward to calculate that the resulting peak shifts are lower than the $E_{F}$ shifts at the surface by $10 \%$ and $60 \%$ for $\lambda=0.8$ and $15 \mathrm{~nm}$, respectively. ${ }^{26}$ For $n^{-}$-InAs the shape of the band bending is more complicated. Numerical integration of the Poisson equation shows that it extends up to $40 \mathrm{~nm}$ into the bulk (see below). The resulting difference between measured peak shifts and $E_{F}$ shift at the surface is $3 \%(20 \%)$ at $\lambda$ $=0.8 \mathrm{~nm}(15 \mathrm{~nm})$.

(2) An adsorbate-induced shift of the final-state energies with respect to the initial-state energies would shift the $k_{z}$ value of an observed transition at selected $h \nu$. This changes the initial state probed, and leads to an apparent energy shift of the peak not due to an $E_{F}$ shift. The resulting error for the $E_{F}$ determination depends on the dispersion of the initial and
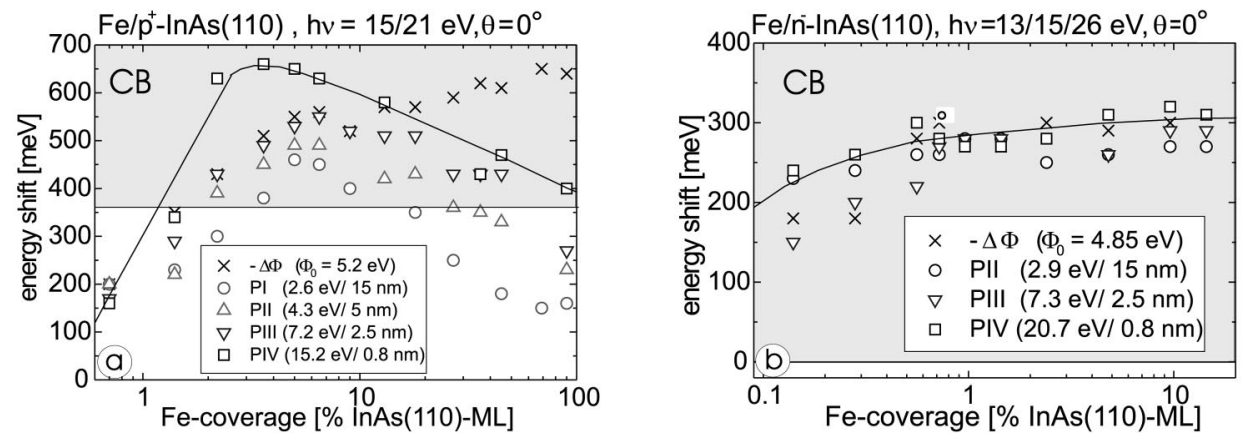

FIG. 4. Peak shifts (open symbols) and negative work-function shifts (crosses) obtained from ARUP spectra at different Fe coverages. The work function measured without $\mathrm{Fe}\left(\Phi_{0}\right)$ and the conduction band $(\mathrm{CB}$, gray) are indicated; photoelectron energies and corresponding mean free paths (Ref. 1) for different peaks are given in brackets; lines are guidelines to the eye; $h \nu$ is indicated above the figure, $\theta=0^{\circ}$ : (a) $p^{+}-\operatorname{InAs}(110)$. (b) $n^{-}-\operatorname{In} A s(110)$. 


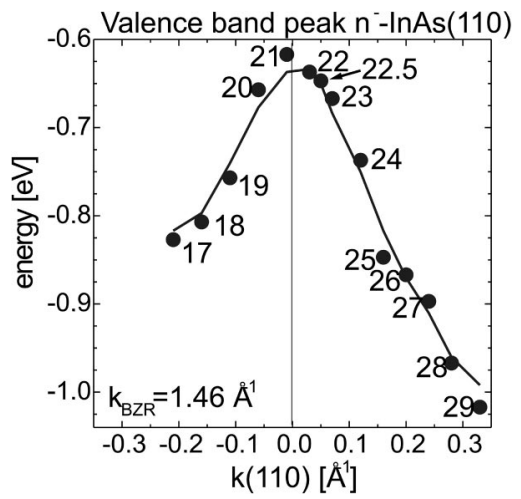

FIG. 5. Peak energies of $P_{I V}$ peaks on $n^{-}$-InAs(110) for different photon energies (given in $\mathrm{eV}$ directly at the data points) $\theta$ $=0^{\circ} . k_{\perp}$ values ( $x$ axis) are obtained by assuming a finite state parabola with the origin at $V_{0}=17 \mathrm{eV} . \mathrm{k}_{B Z R}$ describes the $k_{\perp}$ vector at the edge of the Brillouin zone. The line is a guide to the eye.

final states. Figure 5 shows the measured valence-band dispersion close to the valence-band maximum (VBM). Photon energies are marked, and the $k_{z}$ values at the $x$ axis are obtained by assuming a final-state parabola with an origin at $V_{0}=17 \mathrm{eV}$, determined directly from the photon energy corresponding to the valence-band maximum. Close to the VBM the curve is rather flat, and a $1-\mathrm{eV}$ change of $h \nu$ corresponds to a $40-\mathrm{meV}$ change in the initial-state energy. A change in the energy separation between initial and final states is equivalent to a change in the photon energy. Since it is improbable that the relative energy shift between initial and final states is larger than the absolute $E_{F}$ shift at the surface, we can estimate (from Fig. 5) that the error is less than $4 \%$ close to the VBM. However, for other $k$ points, the dispersion is steeper ${ }^{23}$ and the error could be up to $30 \%$.

With this analysis, we interpret the $P_{I V}$ data in Fig. 4, measured close to the VBM, as the $E_{F}$ shift at the surface reduced by about $10 \%(3 \%)$ for $p^{+}$-InAs $\left(n^{-}\right.$-InAs). For $p^{+}$-InAs $E_{F}$ shifts monotonically upward up to a maximum of $300 \mathrm{meV}$ above the CBM at a coverage of $3.5 \%$ and shifts down to $50 \mathrm{meV}$ above the CBM at a $90 \%$ coverage. For $n^{-}$-InAs, $E_{F}$ also shifts up to $300 \mathrm{meV}$ above the CBM at a coverage of $0.5 \%$, and remains constant up to the highest studied coverage of $12 \%$. Notice that already at the lowest coverage of $0.15 \%$, the $E_{F}$ shift is more than $200 \mathrm{meV}$.

In agreement with the analysis of the influence of $\lambda$, peaks $P_{I}-P_{I I I}$ shift less than $P_{I V}$. Comparison with the mean free path calculation ${ }^{26}$ shows that the observed differences in peak shifts are close to the values expected. In particular the $E_{k i n}$ dependence of the shift is stronger on $p^{+}$-InAs than on $n^{-}$-InAs, reflecting the smaller extension of the band bending on $p^{+}$-InAs. Notice that $P_{I I I}$ is measured with a photon energy probing the steepest region of the valence-band dispersion. ${ }^{23}$ Since $P_{I I I}$ shows nearly the same energy shifts as $P_{I V}$, we can conclude that the error introduced by the adsorbate-induced shifts of final states is of minor importance. From the scatter of the data in Fig. 4, taking into account the different mean free paths of the photoelectrons, we deduce an error bar of about $50 \mathrm{meV}$.

The negative work-function shift $\Delta \Phi$ is also shown in Fig. 4. The work function $\Phi$ itself is measured as the difference between $E_{F}$ and the onset of photoelectron emission. $\Phi$

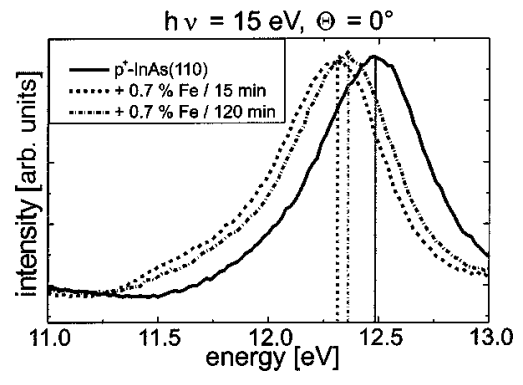

FIG. 6. ARUP spectrum recorded with $0 \%$ and $0.7 \%$ ML Fe on $p^{+}-\operatorname{InAs}(110)$ at the indicated time after deposition; $h \nu=15 \mathrm{eV}$, $\theta=0^{\circ}$, and $E_{\text {pass }}=2.5 \mathrm{eV}$. Vertical lines mark the peaks.

is reduced monotonically up to $90 \%$ coverage. Above $20 \%$ coverage $\Delta \Phi$ clearly differs from the observed $E_{F}$ shift, indicating an adsorbate-induced change in the surface dipole. The measured difference between the $E_{F}$ shift and $\Delta \Phi$ is $130 \mathrm{meV}$ at $36 \%$ coverage and $250 \mathrm{meV}$ at $90 \%$ coverage, which corresponds to a surface dipole of $10^{-30} \mathrm{Cm}$ per $\mathrm{Fe}$ atom. The dipole could be due to positively charged Fe at the surface. Alternatively, the adsorbate could lift the relaxation of the In-As bonds, ${ }^{1}$ thereby annihilating (partly) the original surface dipole of $-2.5 \times 10^{-30} \mathrm{Cm}$ per unit cell. Perhaps both effects take place and contribute to the reduction of the work function.

At low $\mathrm{Fe}$ coverages $(0.7 \%$ and $1.5 \%)$, we observe a distinct back shift of the peaks on $p^{+}-\operatorname{InAs}(110)$ with time. A similar effect was not observed on $n^{-}$-InAs(110). The back shift takes place entirely within the first $2-3 \mathrm{~h}$ after Fe deposition, and is illustrated in Fig. 6. We assume that the effect is related to the diffusion of the $\mathrm{Fe}$ atoms, resulting in the formation of $\mathrm{Fe}$ multimers. About 50 jumps per Fe atom take place within $2 \mathrm{~h}$, implying that $\mathrm{Fe}$ atoms initially up to ten unit cells apart can form dimers on this time scale. Most of the $\mathrm{Fe}$ atoms have neighbors after $2 \mathrm{~h}$. As described below, the formation of dimers significantly reduces the ionization probability of the $\mathrm{Fe}$ atoms, thereby reducing the averaged band bending.

Finally, we describe the ARUPS measurements of the 2DEG. The measured shift of the surface $E_{F}$ into the conduction band leads to band bending and a confinement in the $z$ directon, inducing a 2DEG. If the 2DEG states are occupied they can be measured by ARUPS. ${ }^{15,27}$ Spectral features caused by the 2DEG are found on $n^{-}$-InAs, but not on $p^{+}$-InAs. Figure 7(a) shows a spectrum of $n^{-}-\operatorname{InAs}(110)$ covered by $0.5 \% \mathrm{Fe}$ in comparison with the clean surface. The small peak close to $E_{F}$, only visible in the magnification of the inset, is the 2DEG peak. The 2DEG peak is detected at photon energies $h \nu=10-18 \mathrm{eV}$. At higher $h \nu$ the $P_{I V}$ peak covers the position of the 2DEG peak. Above $h \nu=25 \mathrm{eV}$ the 2DEG peak is no longer covered by $P_{I V}$ (see Fig. 5), but does not reappear. This might be due to the low mean free path of the corresponding photoelectrons $(1 \mathrm{~nm})$ compared with the position of the maximum of the 2DEG density located about $10 \mathrm{~nm}$ below the surface. ${ }^{28}$ Figure 7(b) shows a $3 \mathrm{D}$ representation of the $2 \mathrm{DEG}$ peak as a function of the photoelectron energy and the detection angle $\alpha$. The peak disappears for $\alpha \geqslant 1.5^{\circ}$. This is expected for an InAs 2DEG, since the strong dispersion of the conduction band requires that only $k_{\|}$states close to the Brillouin-zone center are occupied. ${ }^{29}$ 

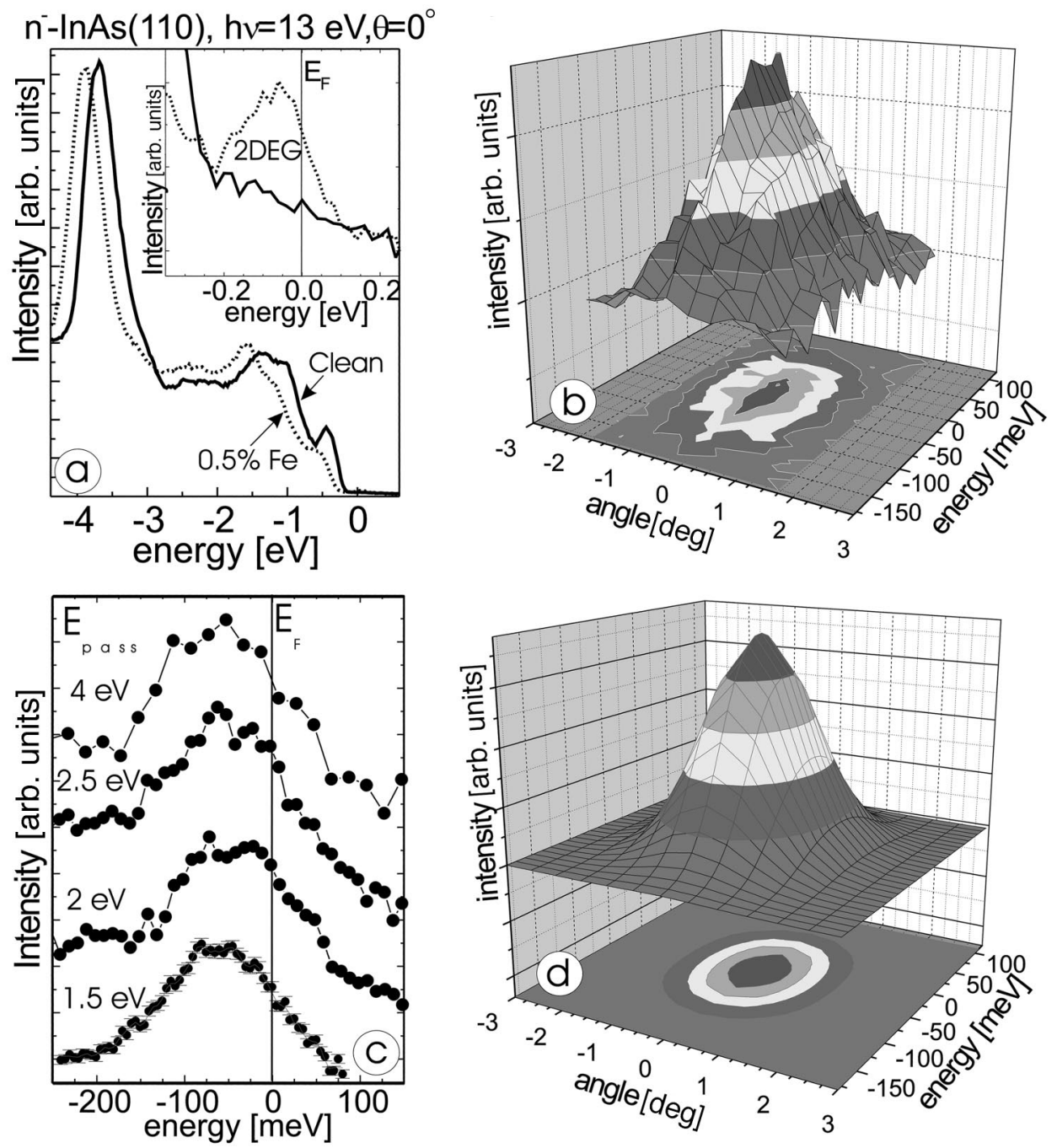

FIG. 7. $0.5 \% \mathrm{ML}$ Fe on $n^{-}-\operatorname{InAs}(110)$. (a) ARUP spectrum recorded at $h \nu=13 \mathrm{eV}, \theta=0^{\circ}$, and $E_{\text {pass }}=2.5 \mathrm{eV}$ in comparison with the clean surface. Inset: magnification of the spectra next to $E_{F}$ showing the 2DEG peak. (b) Three-dimensional representation of the 2DEG spectrum as a function of energy and analyzer angle with respect to normal emission. The bottom image shows a top view contour map of the peak; $h \nu=13 \mathrm{eV}$ and $\theta=0^{\circ}$. (c) $2 \mathrm{DEG}$ spectrum recorded with different $E_{\text {pass }}$ as indicated; $h \nu=13 \mathrm{eV}$ and $\theta=0^{\circ}$. (d) Simulation of the expected spectral intensity of the experiment shown in (b), assuming two subbands at $E_{1}=-70 \mathrm{meV}$ and $E_{2}=-10 \mathrm{meV}$, respectively, and the resolution parameters of the setup.

The 2DEG peak measured, with different energy resolution of the spectrometer $\left(E_{\text {pass }}\right)$, is shown in Fig. 7(c). The full width of half maximum (FWHM) decreases slightly with the spectrometer resolution. However, at $E_{\text {pass }}=1.5 \mathrm{eV}$ the FWHM remains broader than $100 \mathrm{meV}$, and a plateau at the maximum of the peak appears. Since the spectrometer FWHM is $30 \mathrm{meV}$, the photon energy FWHM is $10 \mathrm{meV}$ and the expected thermal broadening FWHM is about 50 $\mathrm{meV}$, the resulting complete "instrumental" FWHM is only $60 \mathrm{meV}$, smaller than the observed peak width. We assume that two subbands cause the broad FWHM of the peak. Notice that lifetime broadening cannot explain the plateau (at the maximum). Assuming two subbands we can reproduce the spectra as shown by the fit in Fig. 7(d) calculated by folding the spectral intensity corresponding to the known InAs dispersion with the "instrumental" FWHM described above and a Gaussian angular resolution of the spectrometer (the FWHM is $1.6^{\circ}$ ). The two subbands are assumed to be at
-70 and $-10 \mathrm{meV}$ below $E_{F}$. The agreement between simulated spectral intensity and experiment is within the experimental error. An independent numerical calculation of the band bending with a surface $E_{F} 300 \mathrm{meV}$ above the CBM (Ref. 33) results in an energy of the first 2DEG subband $65 \mathrm{meV}$ below $E_{F}$, and shows a second subband close to the bulk CBM, $6.5 \mathrm{meV}$ below $E_{F} \cdot{ }^{34}$

\section{DISCUSSION}

There are two generally accepted explanations of the Fermi-level position on adsorbate covered III-V semiconductors. ${ }^{1}$ The first is based on metal-induced gap states (MIGS) and related to the virtual gap states of the complex band structure of the semiconductor. It is applicable to metallic adsorbates with a high density of states at $E_{F} \cdot{ }^{30}$ The resulting $E_{F}$ position depends mainly on the properties of the semiconductor and less on the properties of the adsorbate. For InAs the corresponding $E_{F}$ position is $0.15 \mathrm{eV}$ 
above the CBM. ${ }^{31}$ With some variations $E_{F}=0.15 \mathrm{eV}$ is found after room-temperature deposition of $\mathrm{Cu}, \mathrm{Ag}$, and $\mathrm{Au}$

The 2DEG peak measured, with different energy resolution of the spectrometer $\left(E_{\text {pass }}\right)$, is shown in Fig. $7(\mathrm{c})$. The full width of half maximum (FWHM) decreases slightly with at coverages of more than 20\%. ${ }^{9,32}$ After low-temperature deposition, the same $E_{F}$ is found for coverages above $100 \%{ }^{9}$ It is suspected that metallic islands of the adsorbate are present, if $E_{F}$ is shifted to about $0.15 \mathrm{eV}$. In agreement, we attribute the position of the Fermi level at $90 \%$ Fe coverage to the dominant influence of MIGS.

The other explanation of the Fermi-level shift is applicable to isolated adsorbates and is based on adsorbate induced states of donor type. ${ }^{19}$ The $E_{F}$ position obtained after low-temperature deposition of metallic atoms on $\operatorname{InAs}(110)$ and $\operatorname{GaAs}(110)$ is found to coincide with this model. An intriguing inverse linearity between the ionization energy of the adsorbate and the maximum $E_{F}$ position has been found for both substrates. ${ }^{1,12}$ It was reproduced qualitatively by a simple tight-binding calculation of a local bond between an $\mathrm{sp}^{3}$ orbital of the substrate and an $s$ orbital of the adsorbate. ${ }^{19}$ The maximum position of $E_{F}$ for Fe/InAs(110) is $300 \mathrm{meV}$ above the CBM. We conclude that a donor-type interface state exists $300 \mathrm{meV}$ above the CBM. Using the ionization energy of Fe $(7.87 \mathrm{eV})$, this value fits nicely in the donor energy-ionization energy curve for different adsorbates on InAs(110) given by Aristov et al. ${ }^{12}$

The different coverage dependencies of the two substrates will be analyzed in the light of the surface doping model.,19 The donor levels at the Fe atoms, if located above $E_{F}$, feed electrons to the substrate. The remaining positive $\mathrm{Fe}$ is then surrounded by a screened Coulomb potential inducing a localized band bending. However, each Fe atom (donor level) "feels" the Coulomb potential of neighboring Fe atoms. If the resulting local band bending at the Fe atom is larger than its donor level energy with respect to the $E_{F}$ of the uncovered surface, charging of this Fe atom would cost energy. At the corresponding coverage the band bending comes to saturation. For Fe/InAs(110) this situation is achieved for a band bending of $300 \mathrm{meV}$ above the CBM. So the unperturbed donor level must be $300 \mathrm{meV}$ above the CBM.

The Fe coverage at the maximum of $E_{F}$ is determined by the charge necessary to screen a surface $E_{F}$ located $300 \mathrm{meV}$ above the CBM. ${ }^{1}$ In the case of $p^{+}$-InAs the band bending inhibits all mobile carriers in the depletion region of $13 \mathrm{~nm} .{ }^{1}$ A calculation of the 2DEG states confined in the conduction band (the triangular approximation ${ }^{28}$ ) reveals that the lowest subband of the $2 \mathrm{DEG}$ is $150 \mathrm{meV}$ above $E_{F}$, i.e., it is not occupied. Consequently, the screening is only due to the $\mathrm{Zn}$ acceptors in the depletion region charged by the Fe donors. The areal density of acceptors integrated over the depletion region is $4 \times 10^{-2} / \mathrm{nm}^{2}$. If each $\mathrm{Fe}$ atom charges exactly one acceptor, the band bending would be complete at a coverage of $1 \%$. This coverage is lower than the coverage at maximum $E_{F}$ in our experiment. Ignoring the small differences in $E_{F}$ between $2.1 \%$ and $3.5 \%$ coverages, a coverage of $2.1 \%$ at the maximum of $E_{F}$ remains too high by a factor of 2 . To explain this, the formation of dimers has to be taken into account. It obviously reduces the average band bending (see Fig. 6). The following explanation appears reasonable: $\mathrm{Fe}$ atoms that are close together feel a strong Coulomb potential from a charged neighbor, which reduces their donor level energy below $E_{F}$. Consequently the two atoms are only singly charged.

Next we estimate how many pairs are singly charged at an Fe coverage of $2.1 \%$. The Fe is deposited consecutively, and the first $1.5 \%$ are deposited $3 \mathrm{~h}$ before the ARUPS measurements. On average, they exhibited 90 jumps moving a distance of $9-10$ atomic sites during that time. Fe atoms at $1.5 \%$ coverage are on average eight atomic sites apart directly after deposition. Thus most of these Fe atoms have formed dimers at the time of the measurement. A small (negligible) amount of the last $0.6 \%$ will also have formed dimers within the 15 min between the last deposition step and the measurement.

Assuming that dimers are singly charged, we obtain a charge density of only $1 \%$ at an Fe coverage of $2.1 \%$, explaining the experimentally found position of the maximum. However, making the same argument at $1.5 \%$, we would also obtain a charge density close to $1 \%$. We believe that this indicates that $\mathrm{Fe}$ atoms staying two atomic sites apart are also singly charged. Indeed, using a dielectric constant of $\epsilon$ $=3$, a reasonable estimate for $\epsilon$ at the InAs surface, an Fe atom two sites apart from a charged neighbor feels a Coulomb potential of about $450 \mathrm{meV}$, resulting in a donor level below $E_{F}$. To obtain more quantitative insight into the relation between interatomic distance and charging, further experiments would be useful.

At higher coverages (above 15\%) metallic islands are formed. This is evident from the ARUPS intensity appearing up to $E_{F}$. The resulting occupied MIGS screen the Coulomb potential of the Fe donors, and reduce the averaged $E_{F}$ more and more until it finally fits the charge neutrality level of the virtual induced gap states. ${ }^{31}$ At coverages where metallic islands are not yet formed but the band bending is already 300 $\mathrm{meV}$, the additional $\mathrm{Fe}$ atoms do not change the charge situation resulting in the more or less constant $E_{F}$ between $2 \%$ and $7 \%$ coverages.

On $n^{-}$-InAs, the Fe-donated electrons develop an occupied 2DEG at the surface. This screens the Coulomb potentials of the $\mathrm{Fe}$ atoms. The maximum donated charge is calculated by assuming a homogeneous band bending of 300 $\mathrm{meV}$. Integrating the Poisson equation numerically reveals that the band bending extends over $40 \mathrm{~nm} .{ }^{33}$ The groundstate energy of the 2DEG can be estimated with the help of the triangular approximation ${ }^{28}$ to be $65 \mathrm{meV}$ below $E_{F}$, in accordance with experiment. The first subband contains 8 $\times 10^{11} \mathrm{~cm}^{-2}$ electrons corresponding to a coverage of $0.2 \%$ ionized donor levels. The second subband, which was found in the calculation close to $E_{F}$, does not contribute significantly to the 2DEG charge. Indeed, at the lowest Fe coverage of $0.15 \%, E_{F}$ is already more than $200 \mathrm{meV}$ above the CBM, and at $0.3 \%$ it has nearly reached its maximum position in accordance with the surface doping model. At coverages up to $12 \%$ there is no significant change of $E_{F}$, indicating that formation of metallic islands is negligible and MIGS do not screen. Obviously, Fe dimers play no significant role in these experiments. This is not surprising, since at a coverage of $0.2 \%$, the $\mathrm{Fe}$ atoms are on average $12 \mathrm{~nm}$ apart and cannot find each other during the time of the experiment. Interestingly, the assumption of an unscreened Coulomb potential around each $\mathrm{Fe}$ atom with the dielectric constant of bulk InAs $(\epsilon=14.3)$ results in an attractive electrostatic po- 
tential of only $17 \mathrm{meV}$ at a distance of $6 \mathrm{~nm}$ from the Fe at variance, with an obtained $E_{F}$ shift of $300 \mathrm{meV}$. However, it is to be expected that the surface value $\epsilon$ is significantly less than the bulk value, being about $\epsilon_{\text {surface }} \simeq 2-3$.

\section{SUMMARY}

In summary, we have performed STM and ARUPS measurements on Fe-covered InAs(110). We found that Fe atoms diffuse slowly (one jump in $2 \mathrm{~min}$ ) and anisotropically along the atomic rows of the substrate at room temperature. The adsorbed $\mathrm{Fe}$ atoms induce an additional surface dipole of 1 $\times 10^{-30} \mathrm{Cm}$ per $\mathrm{Fe}$ atom (positive charge away from the surface). Depending on the Fe coverage the Fermi level shifts up to a maximum at $300 \mathrm{meV}$ above the conductionband minimum. The coverage dependence measured on low doped $n$-InAs and highly doped $p$-InAs is explained by the surface doping model. ${ }^{19} \mathrm{We}$ found that the measured (aver- aged) Fermi-level position depends significantly on the spatial distribution of the Fe atoms on the surface; in particular, we suppose that Fe dimers are only singly charged. At coverages above $10 \%$, metallic islands are formed and reduce the Fermi-level position even down to the conduction-band minimum at 1-ML coverage. This is explained by the occupation of metal-induced gap states. ${ }^{1,30}$ The existence of a two-dimensional electron gas is directly proven by ARUPS measurements on Fe-covered $n$-InAs.

\section{ACKNOWLEDGMENTS}

We thank R. Brochier, J. Wiebe, C. Meyer, and A. Wachowiak for technical assistance and helpful discussions. Financial support from SFB 508, DFG (WI 1277/12-1), and Graduiertenkolleg 'Physik nanostrukturierter Festkörper', of the "Deutsche Forschungsgemeinschaft" is gratefully acknowledged.
${ }^{1}$ W. Mönch, Semiconductor Surfaces and Interfaces, Springer Series in Surface Science Vol. 26 (Springer, Berlin, 1993); W. Mönch, Electronic Structure of Metal-Semiconductor Contacts, Perspectives in Condensed Matter Physics Vol. 4 (Kluwer, Milano, 1990).

${ }^{2}$ V. Y. Aristov, G. LeLay, P. Soukiassian, H. Hricovini, J. E. Bonnet, J. Osvald, and O. Olsson, Europhys. Lett. 26, 359 (1994).

${ }^{3}$ V. Yu Aristov, M. Bertolo, P. Altheinz, and K. Jacobi, Surf. Sci. 281, 74 (1993).

${ }^{4}$ Y. Chen, J. C. Hermanson, and G. J. Lapeyre, Phys. Rev. B 39, 12682 (1989).

${ }^{5}$ H. U. Baier, L. Koenders, and W. Mönch, Solid State Commun. 58, 327 (1986); H. U. Baier, L. Koenders, and W. Mönch, J. Vac. Sci. Technol. B 4, 1095 (1986).

${ }^{6}$ T. van Gemmeren, S. Rossi Salmagne, and W. Mönch, Appl. Surf. Sci. 65/66, 625 (1993).

${ }^{7}$ K. Smit, L. Koenders, and W. Mönch, J. Vac. Sci. Technol. B 7, 888 (1989).

${ }^{8}$ V. Yu Aristov, G. LeLay, H. Hricovini, Vinh Le Thanh, and J. E. Bonnet, Phys. Rev. B 47, 2138 (1993).

${ }^{9}$ V. Yu Aristov, Phys. Scr. T39, 333 (1991).

${ }^{10} \mathrm{~W}$. N. Rodrigues and W. Mönch, in Chemistry and Defects in Semiconductor Heterostructures Symposium, edited by M. Kawabe, T. D. Sands, E. R. Weber, and R. S. Williams, MRS Symposia Proceedings No. XI (Materials Research Society, Pittsburgh, 1989), p. 197.

${ }^{11}$ V. Yu Aristov, I. L. Bolotin, and S. G. Gelakhova, Surf. Sci. 251/252, 453 (1991).

${ }^{12}$ V. Yu Aristov, G. LeLay, P. Soukiassian, K. Hricovini, J. E. Bonnet, J. Osvald, and O. Olsson, J. Vac. Sci. Technol. B 12, 2709 (1994).

${ }^{13}$ V. Yu Aristov, P. S. Mangat, P. Soukiassian, and G. LeLay, Surf. Sci. 331-333, 641 (1995).

${ }^{14}$ C. Nowak, J. Krujatz, A. Märkl, C. Meyne, A. Chasse W. Braun, W. Richter, and D. R. T. Zahn, Surf. Sci. 331-333, 619 (1995).

${ }^{15}$ V. Yu Aristov, M. Grekh, V. M. Zhilin, A. Taleb-Ibrahimi, G.
Indlekofer, Z. Hurych, G. LeLay, and P. Soukiassian, Appl. Surf. Sci. 104/105, 73 (1996).

${ }^{16}$ H. S. Karlsson, G. Ghiaia, and U. O. Karlsson, Surf. Sci. 407, L687 (1998).

${ }^{17}$ A. Cabibo, J. R. Childress, S. J. Pearton, F. Ren, and J. M. Kuo, J. Vac. Sci. Technol. A 15, 1215 (1997).

${ }^{18}$ S. Datta and B. Das, Appl. Phys. Lett. 56, 665 (1990).

${ }^{19}$ W. Mönch, Europhys. Lett. 7, 275 (1988).

${ }^{20} \mathrm{Chr}$. Wittneven, R. Dombrowski, S. H. Pan, and R. Wiesendanger, Rev. Sci. Instrum. 68, 3806 (1997).

${ }^{21}$ R. M. Feenstra, J. A. Stroscio, J. Tersoff, and A. P. Fein, Phys. Rev. Lett. 58, 1192 (1987).

${ }^{22}$ Since dangling-bond-related surface bands are located about $1 \mathrm{eV}$ away from the band edges of InAs, it is not straightforward to identify the atomic positions of surface anions and cations from STM images at negative and positive sample biases.

${ }^{23}$ C. B. M. Andersson, J. N. Andersen, P. E. S. Persson, and U. O. Karlsson, Surf. Sci. 398, 395 (1998); C. B. M. Andersson, J. N. Andersen, P. E. S. Person, and U. O. Karlsson, Phys. Rev. B 47, 2427 (1993).

${ }^{24}$ A. Huijser, J. van Laar, and T. L. van Rooy, Surf. Sci. 62, 472 (1977).

${ }^{25}$ D. M. Swanston, A. B. McLean, D. N. McIllroy, D. Heskett, R. Ludeke, H. Munekata, M. Prietsch, and N. J. DiNardo, Surf. Sci. 312, 361 (1994).

${ }^{26}$ J. C Riviere, Surface Analytical Techniques (Oxford University Press, Oxford, 1990), p. 100.

${ }^{27}$ J. M. Layet, M. Carrere, H. J. Kim, R. L. Johnson, R. Belkhou, V. Zhilin, V. Yu Aristov, and G. LeLay, Surf. Sci. 402-404, 724 (1998).

${ }^{28}$ T. Ando, A. B. Fowler, and F. Stern, Rev. Mod. Phys. 54, 437 (1982).

${ }^{29}$ L. O. Olsson, C. B. M. Andersson, M. C. Hakansson, J. Kanski, L. Ilver, and U. O. Karlsson, Phys. Rev. Lett. 76, 2138 (1996).

${ }^{30}$ J. Tersoff, Phys. Rev. Lett. 52, 465 (1984); Phys. Rev. B 30, 4874 (1984).

${ }^{31}$ J. Menendez, Phys. Rev. B 38, 6305 (1988). 
${ }^{31}$ Notice the different meaning of $100 \%$, and $1 \mathrm{ML}$, respectively, in the experiments discussed here and in earlier experiments (Refs. 9 and 12). We refer to the unit cell, since only one adsorptionsite is found, while many experiments refer to the number of surface atoms. For the sake of comparison, we have divided all cover- ages originally referred to the number of surface atoms by two.

${ }^{32}$ R. Seiwatz and M. Green, J. Appl. Phys. 29, 1034 (1958).

${ }^{33}$ V. Yu Aristov, G. LeLay, M. Grekh, V. M. Zhilin, A. TalebIbrahimi, G. Indlekofer, and P. Soukiassian, Surf. Rev. Lett. 2, 723 (1995). 